\title{
EPIDEMIOLOGIA DA DOENÇA DE CHAGAS NA ZONA RURAL DO MUNICÍPIO DE TERESINA-PIAUÍ, BRASIL
}

\author{
Dalva Neves da Costa Bento, Luíz M. Farias, \\ Moacyr F. Godoy e John F. Araújo
}

\begin{abstract}
Na área rural do município de Teresina-Piaui, foram capturados 129 triatomíneos distribuídos em (a) ecótopos arifificiais: habitações (1 Triatoma brasiliensis, 1 Panstrongylus geniculatus, $I$ Rhodnius pictipes $e 1$ Rhodnius prolixus) e galpão abandonado (7 Rhodnius nasutus) e (b) ecótopos naturais: Orbignya martiana (41 Rhodnius neglectus, 33 Rhodnius prolixus $e 41$ Rhodnius nasutus) $e$ Copernicia cerifera ( 3 Rhodnius neglectus). Cerca de 22,6\% dos triatomíneos capturados estavam infectados por flagelados, sendo $30 \%$ no ambiente artificial e 21,9\% no ambiente natural. Dos 28 mamiferos capturados e examinados, 7 Didelphis albiventris, 2 Rattus rattus 1 Tamandua tetradactyla apresentavam-se positivos para flagelados. Os flagelados encontrados nos iriatomineos e nos mamiferos eram semelhantes ao Trypanosoma cruzi. Das 123 reações sorológicas, por imunofluorescência indireta, realizadas na população, duas $(1,6 \%)$ foram reativas.
\end{abstract}

Palavras-chaves: Doença de Chagas. Estudo epidemiológico. Trypanosoma cruzi. Triatomíneos, Piaui.

A doença de Chagas, protozoose primitiva de animais silvestres, resulta de adaptação posterior do parasito ao homem, acometendo hoje cerca de 5 (cinco) milhões de brasileiros, e tornando-se assim, uma das mais graves endemias em nosso País ${ }^{7}$. A transmissão do Trypanosoma cruzi ao homem depende de dois fatores: o desequilibrio ecológico e a má qualidade de habitação do homem, que proporciona assim, a domiciliação dos triatomíneos ${ }^{2}$. $\mathrm{Na}$ profilaxia e controle desta endemia, o conhecimento do ciclo silvestre da doença é de fundamental importância. Sabe-se que espécies de triatomineos, principalmente do gênero Rhodnius, estão altamente adaptados a diferentes tipos de palmeiras ${ }^{123891415}$.

Em Teresina - Piauí, tanto na zona urbana como na rural, predomina em grande quantidade a palmeira "babaçu"(Orbignya martiana), na qual em estudos recentes realizados na área urbana, foi

\footnotetext{
Departamento de Biologia, Centro de Ciências da Natureza e Departamento de Parasitologia e Microbiologia, Centro de Ciências da Saúde, Fundação Universidade Federal do Piauí, Teresina, PI, Brasil e Hospital Santa Maria, Hemodinâmica, Teresinha, PI, Brasil.

Trabalho financiado pela FINEP.

Endereço para correspondência: Dra. Dalva Neves da Costa Bento. Departamento de Biologia/CCN/FUFPI - 64050-900 Teresinha - Piauí.

Recebido para publicação em 19/08/91.
}

constatada a presença de mamíferos e triatomíneos, ambos infectados por Trypanosoma semelhante ao cruzi. As espécies de triatomíneos (Rhodnius nasutus e Triatoma pseudomaculata) e os marsupiais (Didelphis albiventris e Marmosa agilis) capturados na região, parecem desempenhar um importante papel no ciclo deste protozoário, devido ao alto índice de infecção que apresentaram 29,1 e 38,4\%, respectivamente. Por outro lado, a prevalência da infecção chagásica neste município foi de aproximadamente $1,3 \%$, em inquérito sorológico realizado pelo Ministério da Saúde - SUCAM no período $1975 / 81$, sendo esta a única informação disponível sobre a doença no município de Teresina, uma vez que o mesmo não foi incluído ainda no Programa de Controle da Doença de Chagas ${ }^{13}$.

No sentido de dar continuidade aos estudos já iniciados e melhor esclarecer a epidemiologia da doença de Chagas na região, desenvolveu-se o presente trabalho em diferentes localidades das zonas rurais deste município.

\section{MATERIAL E MÉTODOS}

\section{Localidades estudadas}

Os ciclos de transmissão silvestre, peridoméstico 
Bento DNC, Farias LM, Godoy MF, Araújo JF. Epidemiologia da doença de Chagas na zona rural do munictpio de Teresina-Piaui, Brasil. Revista da Sociedade Brasileira de Medicina Tropical 25:51-58, jan-mar, 1992.

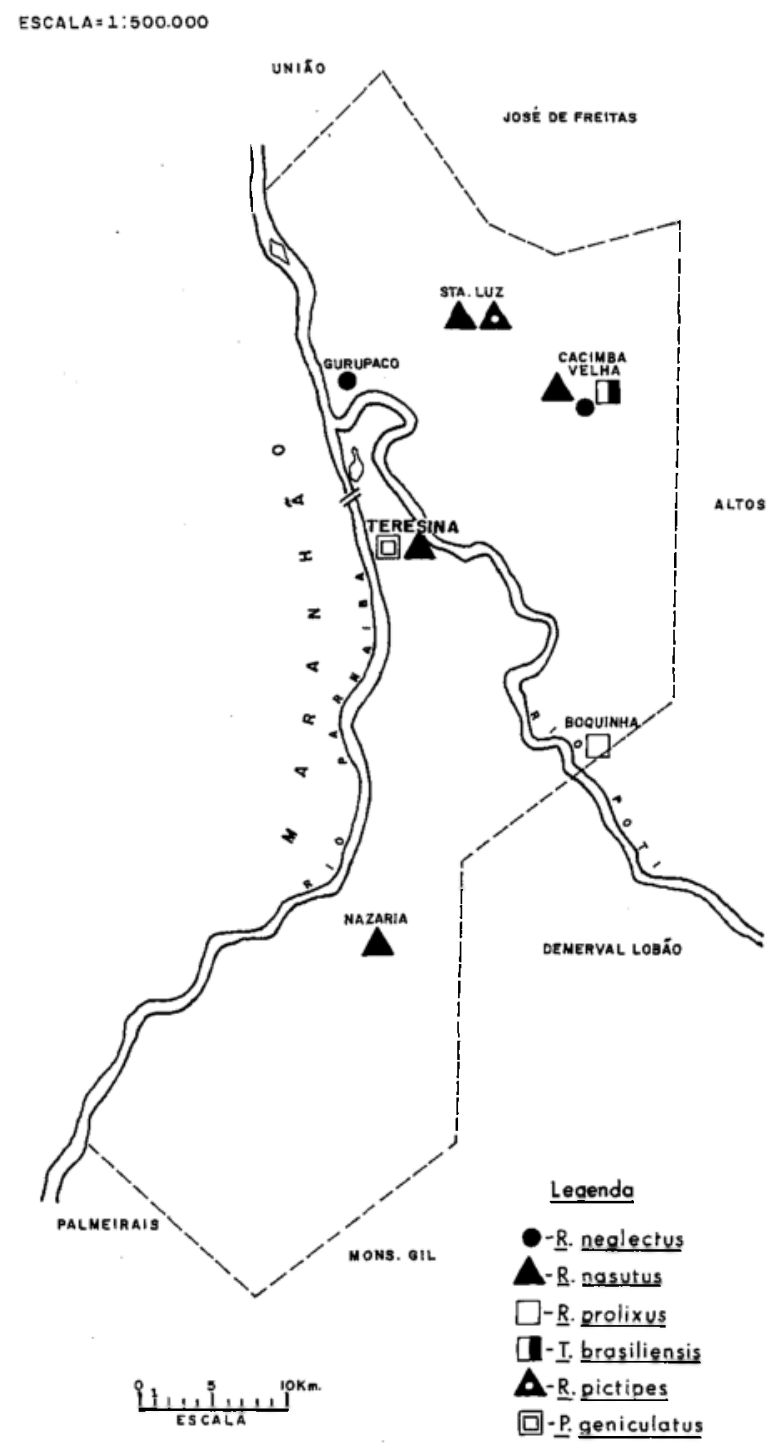

Figura 1 - Distribuição de triatomíneos na área rural de Teresina, Piauí, Brasil - 1986.

e doméstico do $T$. cruzi foram estudados por um período de oito meses (de agosto/85 a março de 1986), nas localidades de Cacimba Velha, Nazária, Boquinha, Gurupaco e Santa Luz, Município de Teresina, Estado do Piauí (Fig. 1).

Estas localidades foram escolhidas levando-se em consideração o critério de maior facilidade de acesso, por via terrestre, aos locais e às casas.

Captura e exame dos triatomíneos

Domicillios e Peridomicílios (Ecótopos Artificiais).
Os triatomíneos foram capturados manualmente com auxílio de insetífugo (solução líquida de piretro $2 \%$ ) nas paredes externas e internas dos domicnios e anexos, estes últimos representados por galinheiros, chiqueiros, apriscos, monte de telhas, madeiras, pedras e cercas.

Ciclo Silvestre (Ecótopos Naturais). A pesquisa de triatomíneos empalmeiras (O. martiana, Copernicia cerifera e Astrocarym sp.) ocorreu após a derrubada das mesmas sobre um pano branco $(5 \mathrm{~m} \times 5 \mathrm{~m})$ as folhas cortadas na base foram cuidadosamente 
Bento DNC, Farias LM, Godoy MF, Araújo JF. Epidemiologia da doença de Chagas na zona rural do município de Teresina-Piaui, Brasil. Revista da Sociedade Brasileira de Medicina Tropical 25:51-58, jan-mar, 1992.

examinadas.

Foram pesquisadas ainda, os ninhos de pássaros e roedores encontrados nas palmeiras, árvores e outros locais.

Os triatomíneos capturados foram identificados e examinados quanto à presença de flagelados no tubo digestivo pela técnica de compressão abdominal. Os triatomíneos negativos, por esta técnica, eram dissecados e examinados. Alguns exemplares foram enviados ao Departamento de Entomologia da FIOCRUZ/Rio de Janeiro, para confirmar a identificação.

Coleta de sangue para sorologia. Foram colhidas amostras de sangue (cerca de $5 \mathrm{ml}$ ), por via endovenosa, de um grupo de 123 pessoas residentes nas casas onde se pesquisava a presença de triatomíneos, sendo 50 do sexo masculino e 73 do sexo feminino, com a idade que variou entre um e noventa anos. Todas as amostras de sangue foram mantidas em condições ambientais, por cerca de 20 horas, para obtenção do soro, que foi conservado à temperatura de $-5^{\circ} \mathrm{C}$, até serem enviados à Fundação Ezequiel Dias (FUNED), em Belo Horizonte -MG, onde foram submetidos a reação de imunofluorescência indireta.

Todos os indivíduos foram examinados clinicamente, em contrapartida para a população.

Os pacientes reativos repetiram exames sorológicos, foram reexaminados clinicamente e submetidos a exames complementares como eletrocardiograma, raio- $\mathrm{X}$ do tórax, hemocultura $\mathrm{e}$ xenodiagnóstico.

Captura, exame e identificação de mamiferos. Para captura de mamíferos silvestres, peridomésticos e domésticos, foram utilizadas armadilhas de vários tipos e tamanhos, industrializadas ou feitas por moradores da região. Os animais de menor porte eram transportados para o laboratório do Departamento de Biologia, para exames quanto à infecção pelo $T$. cruzi .

Os mamíferos silvestres e peridomésticos capturados foram investigados pelo examede sangue a fresco, xenodiagnóstico e hemocultura. Nos mamíferos domésticos (sete gatos, dois porcos, duas cabras e sete cães), foi aplicado apenas o xenodiagnóstico devido às dificuldades de se utilizarem as demais técnicas no campo.
Para realizar os exames, os animais eram imobilizados e, eventualmente, anestesiados com éter.

Coletava-se o sangue da cauda ou veia alar (morcegos) para o exame a fresco e, através da punção cardíaca, para hemocultura, sendo empregado o meio NNN.

No xenodiagnóstico, para cada animal, foram aplicadas 30 ninfas de $4^{\circ}$. estádio dos seguintes triatomíneos: 10 de Triatoma brasiliensis, 10 de Triatoma infestans e 10 de Rhodnius prolixus. O exame dos insetos ocorreu 30 dias após a aplicação do xenodiagnóstico, pela técnica da compressão abdominal, e os negativos eram dissecados e examinados.

\section{RESULTADOS}

\section{Distribuição dos triatomíneos nos ecótopos}

Naturais e Artificiais. A presença de triatomíneos ocorreu em quatro casas $(7,4 \%)$ e em um galpão de granja abandonado $(1,8 \%)$, das 54 unidades domiciliares (domicílio e peridomicúlio) pesquisados.

$O$ índice global de infestação triatomínica nas 12 palmeiras examinadas (nove $O$. martiana, duas C. cerifera e uma Astrocaryum $s p$ ) foi de $75 \%$.

Foram capturados 129 exemplares de triatomíneos, sendo 11 em ecótopos artificiais e 118 em ecótopos naturais. Dos 115 exemplares examinados, 10 de ecótopos artificiais e 150 de ecótopos naturais, 30 e $21,9 \%$ respectivamente, apresentavam-se com infecção natural para tripanosomas semelhantes ao $T$. cruzi.

A Figura 1 mostra a distribuição dos triatomíneos por localidade e a Tabela 1 , os índices de infecção por espécie, distribuídos em seus respectivos ecótopos.

Mamíferos capturados exenodiagnóstico emanimais domésticos e peridomésticos. Dos 28 mamíferos capturados e examinados, $10(35,7 \%)$ apresentaramse positivos por flagelados em uma ou mais das técnicas de diagnóstico utilizadas, sendo sete $D$. albiventris (25\%), dois Rattus rattus $(7,1 \%)$ e um Tamandua tetradactyla $(3,6 \%)$.

A distribuição por espécie, número de 
Bento DNC, Farias $L M$, Godoy MF, Araújo JF. Epidemiologia da doença de Chagas na zona rural do município de Teresina-Piaui, Brasil. Revista da Sociedade Brasileira de Medicina Tropical 25:51-58, jan-mar, 1992.

Tabela 1 - Triatomíneos capturados e infectados por T. cruzi em ecótopos artificiais e naturais, na área rural de Teresina, Estado do Piauí.

\begin{tabular}{|c|c|c|c|c|c|c|c|c|c|}
\hline \multirow{3}{*}{ Ecótopo } & \multicolumn{3}{|c|}{ Adultos } & \multicolumn{3}{|c|}{ Ninfas } & \multicolumn{3}{|c|}{ Totais } \\
\hline & Cap & Exam & Pos & Cap & Exam & Pos & Cap & Exam & Pos \\
\hline & $\mathrm{N}^{0}$ & $\mathrm{~N}^{\circ}$ & $\mathrm{N}^{\circ} \quad \%$ & $\mathrm{~N}^{0}$ & $\mathrm{~N}^{0}$ & $\begin{array}{ll}N^{0} & \%\end{array}$ & $\mathrm{~N}^{0}$ & $\mathrm{~N}^{0}$ & $\begin{array}{ll}\mathrm{N}^{0} & \%\end{array}$ \\
\hline
\end{tabular}

Artificiais:

Intradomiciliar

T. basiliensis

P. geniculatus

R. pictipes

R. prolixus

Peridomiciliar / Galpão

R. nasutus

0

$0 \quad 0$

7

$\begin{array}{llll}4 & 3 & 3 & 100\end{array}$

$\begin{array}{llll}0 & 0 & 0 & 0 \\ 0 & 0 & 0 & 0 \\ 0 & 0 & 0 & 0 \\ 0 & 0 & 0 & 0\end{array}$

Total

$$
4300
$$

7

Naturais:
O. martiana (Palmeira)
R. neglectus
R. prolixus
$R$. nasutus
(2)
C. cerifera (Palmeira)
R. neglectus

$14 \quad 13$

3215,4

27

$15 \quad 15$

1280,0

18

$\begin{array}{llll}4 & 4 & 250\end{array}$

37

1900

$\begin{array}{llll}41 & 32 & 2 & 6,3\end{array}$

$\begin{array}{llll}1 & 1 & 1 & 100\end{array}$

$\begin{array}{llll}2 & 1 & 0 & 0\end{array}$

$\begin{array}{lll}3 & 2 & 1\end{array}$

Total

$\begin{array}{llll}34 & 33 & 17 & 51,5\end{array}$

$84 \quad 72$

68,3

$\begin{array}{lll}118 & 105 \quad 23\end{array}$

21,9

\section{Total}

$\begin{array}{llll}38 & 36 & 20 & 55,5\end{array}$

$91 \quad 79$

67,6

$\begin{array}{llll}129 & 115 & 26 & 22,6\end{array}$

Cap = Capturados

Exam $=$ Examinados

$$
\text { Pos }=\text { Positivos }
$$


Bento DNC, Farias LM, Godoy MF, Araújo JF. Epidemiologia da doença de Chagas na zona rural do município de Teresina-Piaui, Brasil. Revista da Soctedade Brasileira de Medicina Tropical 25:51-58, jan-mar, 1992.

Tabela 2 - Infecção natural em mamiferos capturados na área rural de Teresina - Piauí com flagelados semelhantes ao $\mathrm{T}$. cruzi.

Espécies

Número de

capturados

Número de

examinados

$\frac{\text { Infectados }}{N^{0}} \frac{-}{\%}$

Didelphis albiventris

7

Monodelphis domestica

Galea spixi

Myotis migricans

Molossops sp

Coendu sp

Rattus rattus

Felis sp

Tamandua tetradactyla
7

1

6

1

1

1

9

1

1
7

1

6

1

1

1

9

1

1
7

100

0

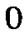

0

0

0

0

$2 \quad 22,2$

0

0

Total

28

28

10

35,7

mamíferos capturados e seus respectivos índices de infecção são encontrados na Tabela 2.

Sorologia (IFI) na população. Em 123 reações sorológicas realizadas na população, duas foram reativas, sendo um indivíduo do sexo masculino e outro do sexo feminino. A sorologia reativa destes pacientes foi confirmada por uma segunda reação. Clinicamente não apresentaram evidências da doença de Chagas (forma indeterminada). Os exames complementares (raio-X e eletrocardiograma) foram normais e o xenodiagnóstico e hemocultura foram negativos.

A Tabela 3 mostra a distribuição dos pacientes por faixa etária, sexo e respectivas sorologias reativas.

\section{DISCUSSÃO}

No Estado do Piauí, o inquérito sorológico, realizado entre 1975/81 pelo Ministério da Saúde, estendeu-se por 114 municípios e a prevalência de infecção chagásica foi estimada em $24 \%$ para todo o Estado ${ }^{4}$. No entanto, o levantamento triatomínico e a borrificação intradomiciliar ficaram restritas a áreas consideradas de alta endemicidade.

Pelo fato de apresentar baixo índice $(1,3 \%)$ de sorologia reativa (Inquérito Nacional), o município de Teresina - Piauí não foi julgado prioritário pelo programa de Controle de Doença de Chagas SUCAM $^{13}$, sendo assim, inexistentes dados sobre a presença de triatomíneos nas áreas rurais deste município. $\mathrm{Na}$ área urbana, o $R$. nasutus e o $T$. pseudomaculata já foram capturados em $95 \%$ e $5 \%$, respectivamente, das $O$. martiana num total de 55 palmeiras examinadas ${ }^{3}$. O $R$. nasutus foi encontrado em $78 \%$ das $C$. cerifera pesquisadas em diferentes municípios do Estado do Piauí ${ }^{15}$.

Investigações semelhantes realizadas na periferia de grandes metrópoles brasileiras descreveram ecótopos naturais e artificiais de triatomíneos e 
Bento DNC, Farias $L M$, Godoy $M F$, Araüjo JF. Epidemiologia da doença de Chagas na zona rural do municipio de Teresina-Piaui, Brasil. Revista da Sociedade Brasileira de Medicina Tropical 25:51-58, jan-mar, 1992.

Tabela 3 - Distribuição por faixa etária da amostra submetida a exame sorológico contra o T. cruzi por imunofluorescência indireta (IFI), no Município de Teresina-Piaui, Brasil.

\begin{tabular}{|c|c|c|c|c|c|c|}
\hline \multirow{2}{*}{$\begin{array}{l}\text { Grupo } \\
\text { Etário }\end{array}$} & \multicolumn{2}{|c|}{ Sexo Masculino } & \multicolumn{2}{|c|}{ Sexo Feminino } & \multicolumn{2}{|c|}{ Total } \\
\hline & $N^{0}$ Sorol & Reat & $\mathrm{N}^{\circ}$ Sorol & Reat & $\mathrm{N}^{\circ}$ Sorol & Reat \\
\hline $0-10$ & 10 & 0 & 13 & 1 & 23 & 1 \\
\hline $11-20$ & 9 & 0 & 14 & 0 & 23 & 0 \\
\hline $21-30$ & 7 & 0 & 14 & 0 & 21 & 0 \\
\hline $31-40$ & 7 & 0 & 13 & 0 & 20 & 0 \\
\hline $41-50$ & 5 & 0 & 8 & 0 & 13 & 0 \\
\hline $51-60$ & 7 & 1 & 8 & 0 & 15 & 1 \\
\hline $61-70$ & 4 & 0 & 1 & 0 & 5 & 0 \\
\hline $71-80$ & 1 & 0 & 2 & 0 & 3 & 0 \\
\hline Total & 50 & 1 & 73 & 1 & 123 & 2 \\
\hline
\end{tabular}

demonstraram ainda, a importância das espécies assinaladas para a doença de Chagas nessas áreas ${ }^{5}$.

No presente estudo, oR. neglectus foi capturado em $C$. cerifera e o $O$. martiana, enquanto o $R$. prolixus e o $R$. nasutus somente em $O$. martiana, já descrita em outros Estados, como domiciliação ${ }^{1}$ 2. A presença de triatomíneos nas palmeiras $O$. martiana e $C$. cerifera sugere a importância destas na manutenção do ciclo silvestre da doença de Chagas no Estado do Piauí devido, principalmente, à sua ampla distribuição por todo o Estado. Por outro lado, o hábito da população de usar folhas na cobertura e paredes das casas poderá facilitar a domiciliação de triatomíneos, que têm como ecótopo natural essas palmeiras, fato já observado por Pifano na Venezuela ${ }^{14}$.

OR. prolixus, espécie considerada anteriormente como sendo estritamente sinantrópica, foi capturado no interior de uma cafua, na localidade Cacimba Velha e em duas palmeiras $O$. martiana na localidade de Boquinha. A cafua na qual foi encontrado o exemplar adulto era coberta com folhas de palmeiras e cercada por $\boldsymbol{O}$. martiana, ocorrendo também, a presença de animais peridomésticos. Ninfas e adultos foram capturados nas duas palmeiras $O$. martiana localizadas aproximadamente a $\mathbf{3 0 0}$ metros do rio Poty e próximas a cafuas e a uma escola. Observações semelhantes e recentes foram demonstradas na Venezuela, onde também esta espécie de triatomíneos pode ser comumente encontrada em biótipos silvestres e sua migração da palmeira para a habitação ocorre com grande intensidade ${ }^{10}$.

O encontro do $R$. prolixus no Piauí (Tabela 1), cuja identificação contou com a colaboração do Prof. Herman Lent ${ }^{12}$, contribuiu para esclarecer controvérsias sobre a presença deste triatomíneo no Nordeste Brasileiro. Deane (1957), estudando exemplares de tipo como $R$. prolixus do Ceará, considerou o $R$. nasutus a única espécie do gênero presente nesta região ${ }^{6}$. Corrobora, no entanto, com o nosso encontro, a confirmação da presença deste triatomíneo em outro Estado, em área limítrofe com 
Bento DNC, Farias LM, Godoy MF, Araüjo JF. Epidemiologia da doença de Chagas na zona rural do município de Teresina-Piaui, Brasil. Revista da Sociedade Brasileira de Medicina Tropical 25:51-58, jan-mar, 1992.

o Nordeste ${ }^{9}$.

A proximidade das casas aos ecótopos naturais e a atração dos triatomíneos pela luz podem explicar o achado exclusivo de adultos de $T$. brasiliensis, $R$. prolixus, $R$. pictipes e Panstrongylus geniculatus no interior dos domicílios, o que poderá proporcionar a colonização destes triatomíneos, exigindo assim mais atenção dos órgãos de saúde pública.

Um percentual de $22,6 \%$ dos triatomíneos capturados em palmeiras e $35,7 \%$ dos mamíferos examinados apresentaram-se infectados por flagelados semelhantes do T. cruzi. Barreto (1979), observou que o alto índice de infecção nos triatomíneos capturados em palmeiras $O$. martiana deve-se à presença de marsupiais, roedores e morcegos infectados neste tipo de ecótopo. Portanto, o encontro de triatomíneos positivos nas palmeiras examinadas sugere a frequente circulação destes mamíferos neste ecótopo, embora não fossem aí capturados. $\mathrm{O} D$. albiventris apresentou maior índice de infecção (100\% na região rural de TeresinaPiauí, confirmado dados de sua importância como reservatório do $T$. cruzi, fato já verificado na área urbana do mesmo município ${ }^{3}$.

Das duas pessoas reativas sorologicamente para o $T$. cruzi, por imunofluorescência indireta, uma era emigrante do Estado do Ceará, com 60 anos de idade, onde poderia ter adquirido a infecção, e o outro, uma criança de 10 anos que nasceu e sempre permaneceu em Nazária, o que sugere ser este um caso autóctone de infecção por $T$. cruzi. Estes pacientes nunca receberam transfusão sanguínea, $o$ que impossibilita admitir a infecção por essa via. Nesta localidade o $R$. nasutus foi encontrado em peridomicílio e, através da população, fomos informados da presença esporádica de barbeiros no interior dos domicílios, fato este não observado por nós.

Estes dados de 1,6\% de sorologia reativa para T. cruzi na população examinada em 6 (seis) localidades do município de Teresina-Piauí referendam os dados do Inquérito Nacional ${ }^{13}(1,3 \%)$.

Apesar da infecção humana ter se revelado excepcional e a invasão de triatomíneos para o interior dos domicílios ser esporádica, os achados de reservatórios naturais e vetores infectados pelo $T$. cruzi, demonstrado no presente trabalho, em estreita relação com a população local, representa uma ameaça epidemiológica em potencial ${ }^{11}$. Chamamos a atenção para as profundas modificações ambientais que vêm ocorrendo nas áreas, com a intensa derrubada de palmeiras destruindo, assim, o ecótopo natural dos triatomíneos e possibilitando, desta forma, a instalação definitiva do ciclo domiciliar na região.

\section{SUMMARY}

In the rural areas of Teresina, 129 triatomines were captured distributed in (a) artificial ecotopes; a house with one Triatoma brasiliensis, one Panstrongylus geniculatus, Rhodnius pictipes, and one Rhodnius prolixus and in a uninhabited chicken house (7 Rhodnius nasutus). (b) Natural ecotopes; Pahus Orbignya martiana (41 Rhodnius neglectus, 33 Rhodnius prolixus and 41 Rhodnius nasutus) and Copernicia cerifera (3 Rhodnius neglectus). The $22.6 \%$ of captured triatomines were infected by flagellates similar to Trypanosoma cruzi. Twenty eight sylvatic mammals were captured and examined. Seven Didelphia albiventris, 2 Rattus rattus and $a$ Tamandua tetradactyla were infected with flagellates. The flagellates found in both triatomines and mammals were morphologically indistinguishable from Trypanosoma cruzi. Serology by the indirect immuno fluorescence test for Chagas disease revealed two positive seroreactions of positivity among 123 inhabitants examined.

Key-words: Chagas disease. Epidemiological studies. Trypanosoma cruzi. Triatomines. Piaui.

\section{AGRADECIMENTOS}

Aos Professores Herman Lent e Teresa Cristina M. Gonçalves pela identificação dos triatomíneos e aos professores Liléia de Diotaiuti e Artur da S. Pinto, pelas sugestões e revisão do tex to. À Fundação Ezequiel Dias, pela realização dos exames de imunofluorescência. A Financiadora de Estudos e Projetos (FINEP) pela concessão de financiamento deste trabalho.

\section{REFERÊNCIAS BIBLIOGRÁFICAS}

1. Alencar EJ, Bezerra OF. Estudos sobre a

epidemiologia da doença de Chagas no Ceará. 
Bento DNC, Farias LM, Godoy MF, Araújo JF. Epidemiologia da doença de Chagas na zona rural do município de Teresina-Piaui, Brasil. Revista da Sociedade Brasileira de Medicina Tropical 25:51-58, jan-mar, 1992.

XXIV. Ecologia de triatomíneos do município de Crato, triatomíneos de palmeiras. In: Resumos do $\mathrm{V}$ Congresso Brasileiro de Parasitologia, Rio de Janeiro. p. $29,1980$.

2. Barreto MP. Epidemiologia. In: Brener Z, Andrade $\mathrm{Z}$ (ed) Trypanosoma cruzi e Doença de Chagas. $1^{\mathrm{a}}$ edição, Guanabara-Koogan, Rio de Janeiro p. 89$151,1979$.

3. Bento DNC, Castelo Branco AZCL, Freitas MR, Pinto AS. Epidemiologia studies of Chagas disease in the urban zone of Teresina. State of Piauí, Northeastern Brasil. Revista da Sociedade Brasileira de Medicina Tropical 17:199-203, 1984.

4. Camargo ME, Silva GR, Castilho EA, Silveira AC. Inquérito sorológico da prevalência de infecção chagásica no Brasil. 1975/1980. Revista do Instituto de Medicina Tropical de São Paulo 26:192-204, 1984.

5. Coura JR, Ferreira LF, Silva JC. Triatomíneos no Estado da Guanabara é suas relações como domicilio humano. Revista do Instituto de Medicina Tropical de São Paulo 8:162-166, 1966.

6. Deane LM, Deane MP. Notas sobre transmissores e reservatórios do Trypanosoma cruzi no Noroeste do Estado do Ceará. Revista Brasileira de Malariologia e Doenças Tropicais IX:577-595, 1957.

7. Dias JCP. Control of Chagas Disease in Brazil, Parasitology Today 3:336-341, 1987.

8. Diotaiuti L, Dias JCP. Ocorrência e Biologia do Rhodnius neglectus. Lent, 1954 em Macambeiras da periferia de Belo Horizonte, Minas Gerais. Memórias do Instituto Oswaldo Cruz 79:293-301, 1984.

9. Diotaiuti L, Silveira AC, Elias M. Sobre o encontro de Rhodnius prolixus Stal 1859, em macaubeiras. Revista Brasileira de Malariologia e Doenças Tropicais 36:11-14, 1984.

10. Gamboa J. La población silvestre de Rhodnius prolixus em Venezuela. Archivos Venezuelanos Medicina Tropical y Parasitologia Medica 5:321352, 1973.

11. Hoare CA. Reservoir host and natural foci of human protozoal infections. Acta Tropica 19:281-317, 1962.

12. Lent $H$, Wygodzinski P. Revision of the triatominae (hemiptera, reduviidae), and their significance as vectors of Chagas disease. Bulletin of the American Museum of Natural History, New York, 163: 1979.

13. Ministério da Saúde (SUCAM) - Diretoria Regional do Piauí, 1981.

14. Pifano FC. La dinamica epidemilogia de la enfermedad de Chagas en el Valle de los Narnojos, Estado de Carabobo, Venezuela I - Contribución al estudio de los focos naturales silvestres de Schizotrypanum cruzi Chagas, 1909. Archivos Venezuelanos de Medicina Tropical y Parasitologia Médica 5:3-29, 1973.

15. Pinto AS, Bento DNC. The palm tree Copernicia cerifera (carnaúba) as an ecotope of Rhodnius nasutus in rural areas of the state of Piauí Northeastern Brazil. Revista da Sociedade Brasileira de Medicina Tropical 19:243-245, 1986. 